\title{
Paraplegia
}

\section{Effect of Respiratory Training with a Mouth-nose-mask in Tetraplegics}

\author{
F. Biering-Sørensen, MD, PhD, J. Lehmann Knudsen, MD, A. Schmidt, PhD, \\ A. Bundgaard, MD, PhD, I. Christensen, PT \\ Centre for Spinal Cord Injured, Department TH, and Laboratory of Respiratory \\ Physiology, Department of Internal Medicine B, Rigshospitalet, University Hospital, \\ Denmark.
}

\begin{abstract}
Summary
Ten tetraplegics, 8 males and 2 females, with a median age of 32 years participated in a scheduled 6 weeks training programme with a respiratory muscle training mouth-nosemask (RMT-mask) with a fixed expiratory and an increasing inspiratory resistance set by the tetraplegic in accordance to his/her increasing ability during the training period. During the 6 weeks the tetraplegics required to use the RMT-mask for 15 minutes three times a day. Before and after each training session they measured peak flow (PEF). Lung volumes, ventilatory and diffusion capacity were measured before and after the 6 weeks training period. The training resulted only in a significant change in the PEF, which increased with $11 \%$ from $371 \mathrm{l} / \mathrm{min}$ before to $412 \mathrm{l} / \mathrm{min}$ in average after the 6 weeks of training $(p<0.025)$. This statistically significant increase was confirmed by the measurements of PEF performed by the tetraplegics themselves during the training period. In addition there was an increase in PEF from before to immediately after each 15 minutes training session, this trend reached statistically significance $(p<0.025)$ in the third ' 2 weeks period'. These results might indicate a possibility of improving the tetraplegics ability to cough by use of a simple RMT-mask, which in turn might prevent certain lung complications including pneumonia, and atelectasia.
\end{abstract}

Key words: Tetraplegia; Respiratory training; Lung function; Peak expiratory flow.

In patients with tetraplegia the work of breathing is increased due to a reduction of compliance, and because most of the rib cage musculature is paralysed (FuglMeyer, 1971, Gross et al., 1980). This causes a high incidence of respiratory morbidity and mortality (Bellamy et al., 1973, Botterell et al., 1975, Cheshire and Coats, 1966, Fine et al., 1986, Nyqvist and Bors, 1967/1968, Silver and Gibbon, 1968). The risk of atelectasia and pneumonia remain high for these patients throughout their lives, primarily due to difficulty in eliminating bronchial secretions, not least because of a poor cough (Siebens et al., 1964).

Correspondence to: F. Biering-Sørensen, Rigshospitalet, Havnevej 25, DK-3100 Hornbæk, Denmark. 
The aim of the present study was to evaluate if respiratory muscle training by use of a mouth-nose-mask could improve some of the lung parameters in chronic tetraplegics.

\section{Patients and Methods}

Eighteen adult wheelchair-bound tetraplegics were invited to participate in the study. Of these only 10 eventually took part in the respiratory muscle training after informed consent. Basic data for the participating tetraplegics are given in Table I. In addition their median weight was $68 \mathrm{~kg}$ (range 49-90 kg), and their median height $179 \mathrm{~cm}$ (range 168-186 cm). Lung roentgenograms and ECG were found normal at the entry into the study and chest physical examination revealed no signs of respiratory disease at the time of the study.

The respiratory muscle training was scheduled to last 6 weeks, and was carried out by the use of a respiratory muscle training mouth-nose-mask (RMT-mask), to be used for 15 minutes three times a day. The expiratory as well as the inspiratory resistance could be set individually on the RMT-mask.

Before and after the scheduled 6 weeks of respiratory training the following lung function parameters were measured while the tetraplegics remained seated in their wheelchair: lung volumes [forced expiratory vital capacity (EVC); functional residual capacity (FRC); residual volume (RV); and total lung capacity (TLC)] were measured by the closed circuit helium dilution method-the best of three readings of the EVC was used. Forced expiratory volume in one second $\left(\mathrm{FEV}_{1}\right)$ was measured by a Bernstein spirometer, and peak expiratory flow (PEF) by Wrights peak flow meter. Maximal expiratory flow at $50 \%$ and $25 \%$ of EVC $\left(\mathrm{MEF}_{50}\right.$ and $\left.\mathrm{MEF}_{25}\right)$ was calculated from flow-volume curves obtained from an Ohio rolling seal spirometer-the best of three readings of the forced expiratory flows was used. Nitrogen wash-out time was measured by a Hewlett-Packard 47302A Nitrogen analyser, taking $2 \%$ nitrogen as indication for total wash-out. Diffusion capacity was measured by a Morgan transfer test, the best of two correct tries was used. Completion of these lung function measurements took 45-60 minutes. All the measured values were compared to available normal values in the laboratory for persons of same gender, age, and height.

In connection with the lung function test prior to the training period the patients were instructed in the correct use of a Wright mini peak flow meter. Before and after each 15 minutes training session with the RMT-mask, the PEF was measured with this equipment.

Before the training period began, the tetraplegic patients were individually instructed by a physiotherapist in the use of the RMT-mask, and the physiotherapist also decided whether the tetraplegic could manage the training alone or if he needed someone to hold the mask tight against the face during the training sessions. At this primary instruction a fixed expiratory resistance (diameter 4.5 $\mathrm{mm}$ ) was set. The training was always carried out in the sitting position.

The training period started with the smallest inspiratory resistance in the mask, i.e. corresponding to a diameter of $5 \mathrm{~mm}$. When three daily training sessions of 15 minutes each could be carried through with this resistance it was kept constant for 2 days. Then the resistance was increased, i.e. the inspiratory diameter was decreased by $0.5 \mathrm{~mm}$. If the training sessions could not be completed with this 


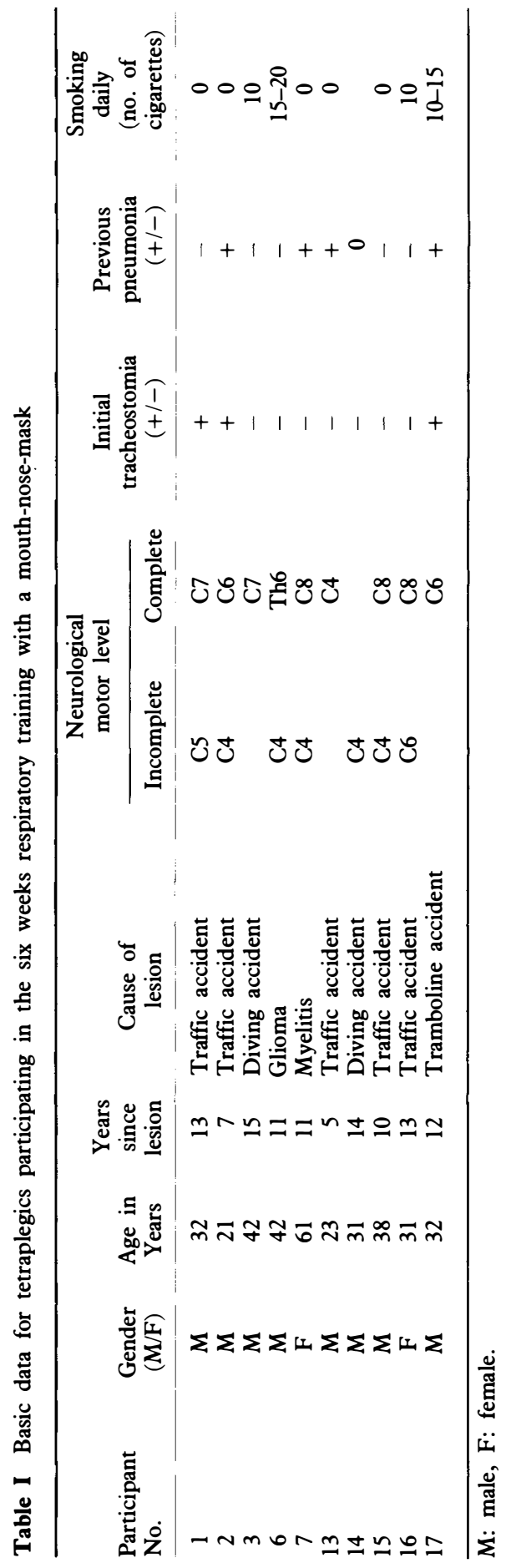


increased resistance the last part of the session was performed with the previous resistance. On the other hand when the three daily sessions could be completed with the new resistance the procedure was repeated until the training session could be carried out, if possible, with the maximal inspiratory resistance, i.e. corresponding to a diameter on $1.5 \mathrm{~mm}$. Following the first week of training the physiotherapist controlled if the training was performed correctly. The actual inspiratory resistance is partly determined by the patients inspiratory flow. During inspiration the negative pressure developed in the system could have been measured and related to the most negative pressure the patient at that lung volume could develop during a maximal inspiration. This was not done, but might prove useful in future studies. In this study endurance on a given resistance was used.

During the whole training period the patient filled out a form each day giving the measurements of PEF before and after each 15 minute training session, the inspiratory resistance used, minutes of training, and if there was a cough and sputum production. Also subjective remarks regarding breathing etc.

Statistical comparisons were carried out with Students t-test, and 5\% was chosen as level of significance.

\section{Results}

The results of the lung function measurements before and after the 6 weeks training period can be seen in Table II.

All parameters except PEF remained virtually unchanged following the training period. PEF increased by $11 \%$ (from 371 to $412 \mathrm{l} / \mathrm{min}$ ), which is a statistically significant increase $(p<0.025)$. This increase was confirmed by the measurements of PEF carried out by the patients themselves (Table III). The increase from the first to the third two week period of training was statistically significant $(p<0.02)$ both for the PEF measurements performed before and after the 15 minutes of training. In addition the recordings in Table III show an increase in PEF from before to after the 15 minute training period, and this increase reached the level of significance in the third two week period $(p<0.025)$. It can be noted that this increase is primarily due to the tetraplegics, who smoked daily.

Table II Average of lung function parameters for 9 tetraplegics before and after a 6 weeks training period with a respiratory muscle training mouth-nose-mask. Absolute values as well as percentage of expected normal values for persons of same gender, age and height are indicated

\begin{tabular}{|c|c|c|c|c|}
\hline & $\begin{array}{l}\text { Before } \\
\text { training }\end{array}$ & $\begin{array}{l}\text { Pct. of } \\
\text { normal }\end{array}$ & $\begin{array}{c}\text { After } \\
\text { training }\end{array}$ & $\begin{array}{l}\text { Pct. of } \\
\text { normal }\end{array}$ \\
\hline Vital capacity (l) & $2 \cdot 41$ & $54 \cdot 6$ & $2 \cdot 40$ & $54 \cdot 2$ \\
\hline Functional residual capacity (l) & $3 \cdot 26$ & $93 \cdot 4$ & $3 \cdot 31$ & $95 \cdot 0$ \\
\hline Residual volume (l) & $2 \cdot 73$ & $164 \cdot 2$ & $2 \cdot 80$ & $168 \cdot 6$ \\
\hline Total lung capacity (I) & $5 \cdot 14$ & $85 \cdot 8$ & $5 \cdot 20$ & $86 \cdot 7$ \\
\hline Forced expiratory volume in the first second (l) & $2 \cdot 17$ & $61 \cdot 2$ & $2 \cdot 19$ & $61 \cdot 7$ \\
\hline Maximal expiratory flow at $50 \%$ of vital capacity $(1 / \mathrm{sec})$ & $2 \cdot 87$ & $62 \cdot 1$ & $3 \cdot 03$ & $65 \cdot 7$ \\
\hline Maximal expiratory flow at $25 \%$ of vital capacity $(1 / \mathrm{sec})$ & $2 \cdot 42$ & & $2 \cdot 40$ & \\
\hline Peak expiratory flow $(1 / \mathrm{min})$ & $371^{\star}$ & $66 \cdot 3$ & $412^{\star}$ & $73 \cdot 9$ \\
\hline Nitrogen wash-out time (min) & $3 \cdot 73$ & $112 \cdot 4$ & $3 \cdot 79$ & $114 \cdot 0$ \\
\hline Total CO-diffusion capacity $(\mathrm{mmol} / \mathrm{min} / \mathrm{kPa})$ & $8 \cdot 23$ & $74 \cdot 0$ & $7 \cdot 90$ & $71 \cdot 1$ \\
\hline CO-diffusion $/ \mathrm{l}$ alveolar volume $(\mathrm{mmol} / \mathrm{min} / \mathrm{kPa} / \mathrm{l})$ & $1 \cdot 81$ & $101 \cdot 7$ & $1 \cdot 78$ & $99 \cdot 9$ \\
\hline
\end{tabular}

${ }^{\star} \mathrm{p}<0.025$. 
Table III Average of individual peak expiratory flow values $(\mathrm{l} / \mathrm{min})$ before and after 15 minutes respiratory muscle training with a mouth-nose-mask. Period 1 is the first 2 weeks of the total training period of 6 weeks and period 3 is the last 2 weeks of the total training period. $\mathrm{n}$ is the number of 15 minutes training sessions completed in the period

\begin{tabular}{|c|c|c|c|c|c|c|}
\hline \multirow{2}{*}{$\begin{array}{l}\text { Subject } \\
\text { no. }\end{array}$} & \multicolumn{3}{|c|}{ Period 1} & \multicolumn{3}{|c|}{ Period 3} \\
\hline & $\mathbf{n}$ & before & after & $\mathbf{n}$ & before & after \\
\hline 1 & 26 & 478 & 484 & 38 & 488 & 497 \\
\hline 2 & 36 & 266 & 257 & 42 & 279 & 273 \\
\hline 3 & 40 & 365 & 388 & 6 & 375 & 377 \\
\hline 6 & 40 & 489 & 496 & 40 & 497 & 505 \\
\hline 7 & 42 & 333 & 320 & 42 & 354 & 357 \\
\hline 13 & 39 & 250 & 251 & 23 & 299 & 300 \\
\hline 14 & 41 & 347 & 347 & 38 & 339 & 352 \\
\hline 15 & 40 & 437 & 433 & & & \\
\hline 16 & 41 & 420 & 436 & 28 & 483 & 493 \\
\hline 17 & 41 & 388 & 399 & 36 & 426 & 436 \\
\hline Average & 39 & 375 & 379 & 33 & 396 & 401 \\
\hline
\end{tabular}

The number of 15 minute training sessions completed for the ten participating tetraplegics was 111 in the median (range 68-124) out of 126 maximal possible, i.e. 3 per day in 6 weeks. Excluding participants nos. 3 and 15 the median was 118 sessions (range 94-124).

Registration of cough and sputum production revealed that only participant no. 6 , who was the heaviest smoker, suffered from this during the training period.

In an interview $1 / 2-1$ year after completion of the training period six of the tetraplegics said that they found the training sessions exhausting, and the 2 female participants were not able to complete training sessions with the highest resistance (diameter $1.5 \mathrm{~mm}$ ). Four of the tetraplegics found that breathing was easier during the training period. The lung function measurements revealed no difference between these four and the others. However, three of the tetraplegics were given, after their own choice, a RMT-mask so that they could continue the training after the study had terminated.

\section{Discussion}

It has been shown that lung function measurements are as reproducible in tetraplegics as in persons with normal respiratory musculature (unpublished data).

The present results on lung function parameters in Table II confirm several previous investigations in tetraplegics (Bergofsky, 1964, Braun et al., 1982, Cameron et al., 1955, Forner, 1980, Fugl-Meyer, 1971, Fugl-Meyer and Grimby, 1971, Haas et al., 1985; Huldtgren et al., 1980, Ledsome and Sharp, 1961, Maloney 1979, McKinley et al., 1969, Ohry et al., 1975, Stone and Keltz, 1963) demonstrating a pronounced restrictive ventilatory impairment, not least caused by altered mechanical properties of the chest wall as a result of the paralysis (Fugl-Meyer and Grimby, 1984).

The training performed with the RMT-mask, increased the PEF significantly, but did not change any of the other measured lung function parameters. This is 
somewhat unexpected, because the training aimed at increasing inspiratory muscle strength and in particular the diaphragm was expected to be trained. In addition we found no increase in the EVC. This does not exclude an increase in the inspiratory vital capacity (IVC), which we did not measure. An increase in IVC could be the background for the improved PEF, due to an increased elastic recoil when the vital capacity is larger. Furthermore the accessory respiratory muscles probably have been strengthened during the RMT-training and hereby contributed to the increase of PEF. The increase in PEF we found on $11 \%$ in average is nearly as high as the improvement in the expiratory flow demonstrated by Braun et al. (1984) in the study of abdominal push assistance during cough. The increased PEF might indicate that a more sufficient cough will be possible (Siebens et al., 1964, Braun et al., 1984), thus improving the tetraplegic's ability to eliminate brochial secretion which might prevent some respiratory complications, in accordance with the impression of Fugl-Meyer and Grimby (1984). The training programme the tetraplegics were asked to carry out was very demanding, and almost all the participating tetraplegics remarked on this. Eight of the invited tetraplegics found the programme too demanding, and did not want to take part in the study. Still it should be noted that 3 of the 10 tetraplegics who completed the study wished to continue using the RMT-mask. It should also be remarked that although none of the tetraplegics completed the respiratory training fully, we found the significant increase in PEF. To find the minimal amount of training necessary to be beneficial will of course need further studies. Gross et al. (1980) found an increased endurance and strength of the diaphragm during inspiratory muscle training of 15 minutes twice daily.

They also found that training at a higher load gave a further increase in endurance. In the study of Gross et al. (1980) only two inspiratory resistances were used for a longer period, whilst we in accordance with the tetraplegic's subjective ability gave them the possibility to increase the inspiratory load through eight resistances. Like our study, Gross et al. (1980) found symptomatic improvement from the respiratory training.

Even though our study did not include a control group we feel that our results justify us to advise tetraplegic patients who experience repeated pneumonia to perform respiratory training as we describe, with inspiratory resistance. The training intensity does not necessarily have to be as high as in the present study.

\section{Acknowledgement}

The RMT-masks were supplied by Astra Meditec.

\section{References}

Bellamy R, PITTS FW, StaufFer ES 1973 Respiratory complications in traumatic quadriplegia. Fournal of Neurosurgery 39:596-600.

BERGOFSKY EH 1964 Mechanism for respiratory insufficiency after cervical cord injury. Annals of Internal Medicine 61:435-446.

BotTerell EH, Jousse AT, Kraus AS, Thompson MG, Wynne-Jones M, Geisler WO 1975 A model for the future care of acute spinal cord injuries. Canadian fournal of Neurological Science 2:361-380.

BRAUn SR, GIOVANNONi R, O’ConNOR M 1984 Improving the cough in patients with spinal cord injury. American foumal of Physical Medicine 63:1-10. 
BRAUN SR, GIOvanNoni R, LEvin AB, HARVEy RF 1982 Oxygen saturation during sleep in patients with spinal cord injury. American foumal of Physical Medicine 61:302-309.

CAMERoN GS, SCOTt JW, Jousse AT, BotTEREll EH 1955 Diaphragmatic respiration in the quadriplegic patient and the effect of position on his vital capacity. Annals of Surgery 141:451-456.

CheshiRe DJE, CoATEs DA 1966 Respiratory and metabolic management in acute tetraplegia. Paraplegia 4:1-23.

Fine PR, DeVivo MJ, Go BK, Lazarus PB, Kartus PL, Rutt RD, Stover SL 1986 The state of the national SCI database. Paraplegia 24:51-52.

FORNER JV 1980 Lung volumes and mechanics of breathing in tetraplegics. Paraplegia 18:258-266.

FUGL-MEYER AR 1971 Effects of respiratory muscle paralysis in tetraplegic and paraplegic patients. Scandinavian foumal of Rehabilitation Medicine 3:141-150.

FUGL-MEYER AR, GRIMBY G 1971 Ventilatory function in tetraplegic patients. Scandinavian fournal of Rehabilitation Medicine 3:151-160.

FUGL-MEYER AR, GRIMBY G 1984 Respiration in tetraplegia and in hemiplegia: a review. International Rehabilitation Medicine 6:186-190.

Gross D, LADD HW, RILEY EJ, MACKLEM PT, GRASSINO A 1980 The effect of training on strength and endurance of the diaphragm in quadriplegia. American foumal of Medicine 68:27-35.

HaAs F, Axen K, Pineda H, Gandino D, HaAs A 1985 Temporal pulmonary function changes in cervical cord injury. Archives of Physical Medicine and Rehabilitation 66:139-144.

HuldTGRen A-C, Fugl-Meyer AR, JonASSON E, BAKE B 1980 Ventilatory dysfunction and respiratory rehabilitation in post-traumatic quadriplegia. European fournal of Respiratory Diseases 61:347-356.

LEDSOME JR, SHARP JM 1961 Pulmonary function in acute cervical cord injury. American Review of Respiratory Diseases 124:41-44.

MALONEY FP 1979 Pulmonary function in quadriplegia: Effects of a corset. Archives of Physical Medicine and Rehabilitation 60:261-265.

MCKinley AC, Auchincloss JR JH, Gilbert R, Nicholas JJ 1969 Pulmonary function, ventilatory control, and respiratory complications in quadriplegic subjects. American Review of Respiratory Diseases 100:526-532.

NyQvist RH, BoRs E 1967/1968 Mortality and survival in traumatic myelopathy during nineteen years, from 1946 to 1965. Paraplegia 5:22-48.

OHRY A, Molho M, RozIN R 1975 Alterations of pulmonary function in spinal cord injured patients. Paraplegia 13:101-108.

SiEBENS AA, KIRBY NA, Poulos DA 1964 Cough following transection of spinal cord at C-6. Archives of Physical Medicine and Rehabilitation 45:1-8.

SILVER JR, GIBBON NOK 1968 Prognosis in tetraplegia. British Medical fournal 4:79-83.

STONE DJ, KeLTZ H 1963 The effect of respiratory muscle dysfunction on pulmonary function. Studies in patients with spinal cord injuries. American Review of Respiratory Diseases 88:621-629. 